\title{
Fluctuation-Induced Casimir Forces in Granular Fluids
}

\author{
C. Cattuto, ${ }^{1,2}$ R. Brito, ${ }^{3}$ U. Marini Bettolo Marconi,${ }^{4}$ F. Nori, ${ }^{2,5}$ and R. Soto ${ }^{3,6}$ \\ ${ }^{1}$ Museo Storico della Fisica e Centro Studi e Ricerche "Enrico Fermi”, Compendio Viminale, 00184 Roma, Italy \\ ${ }^{2}$ Frontier Research System, The Institute of Physical and Chemical Research (RIKEN), Saitama, 351-0198, Japan \\ ${ }^{3}$ Departamento de Física Aplicada I and GISC, Universidad Complutense, 28040 Madrid, Spain \\ ${ }^{4}$ Dipartimento di Fisica, Università di Camerino, 62032 Camerino, Italy \\ ${ }^{5}$ Physics Department and MCTP, The University of Michigan, Ann Arbor, Michigan 48109-1040, USA \\ ${ }^{6}$ Departamento de Física, FCFM, Universidad de Chile, Casilla 487-3, Santiago, Chile
}

(Received 30 November 2005; published 1 May 2006)

\begin{abstract}
We numerically investigate the behavior of driven noncohesive granular media and find that two fixed large intruder particles, immersed in a sea of small particles, experience, in addition to a short-range depletion force, a long-range repulsive force. The observed long-range interaction is fluctuation-induced and we propose a mechanism similar to the Casimir effect that generates it: The hydrodynamic fluctuations are geometrically confined between the intruders, producing an unbalanced renormalized pressure. An estimation based on computing the possible Fourier modes explains the repulsive force and is in qualitative agreement with the simulations.
\end{abstract}

DOI: 10.1103/PhysRevLett.96.178001

PACS numbers: 45.70.- n, 05.40.- a

Granular materials have been extensively investigated because of their complex dynamics [1]. Examples of this include pattern formation, Faraday waves, avalanches, convection phenomena, segregation, and many more. One of the most active fields is the behavior of granular mixtures, e.g., the Brazil nut effect [2] and its multiple variations [3]. In these experiments, the granular material is composed of a mixture of two types of particles differing in mass or size. When the system is agitated, particles of different types may group together (demixing) or stay mixed [4]. Phase diagrams for the mixing or demixing transitions have been constructed for different material properties or experimental conditions. However, a fundamental question remains unanswered: Is the mixing or demixing caused by an effective long-range force between the particles? If so, which is its origin? Let us note that a long-range force is difficult to justify a priori, as grains interact only through a short-ranged hard-core potential.

Recently, three experiments on driven granular mixtures [5-7], performed under very different experimental conditions, give a hint on how to answer the previous question. These experiments have shown that thermodynamic properties (such as pressure [5], density [6], and velocity fluctuations [7]) are different in the regions between the larger particles, versus the remaining, external, regions. Consequently, the big particles modify some physical properties in the confined area between them. The most likely reason is that the larger particles limit the allowed wave vectors of the hydrodynamic fluctuations of the small particles that surround the larger ones.

Forces arising from the confinement of a fluctuation spectrum have attracted attention since the seminal work in 1948 of Casimir, who predicted the existence of an attractive force between two metal plates separated by the vacuum, due to constraints on the quantum electromag- netic field in the gap imposed by the conducting plates [8]. In fact, the concept of Casimir force is more general and is common to systems characterized by long-range fluctuations subject to a geometrical constraint which limits the long-wavelength portion of their spectrum. Soft condensed matter provides examples of Casimir forces, such as those arising in confined critical fluids, liquid crystals, and superconducting films, where long-range correlations are the consequence of a broken continuous symmetry [9]. Vibrated granular matter [10] and granular avalanches [11] provide other examples of physical systems where correlations can become long-ranged, in spite of having shortrange forces.

In this Letter, we show that there is an effective longrange force between the large and heavy particles in a granular mixture. Two ingredients are required: (i) longrange correlations and (ii) the confinement of the fluctuation spectrum induced by the large particles in the density, velocity, and temperature fields.

We consider the driven granular model of Refs. [10,12]. Grains are hard particles of diameter $d$ and mass $m$, and their collisions are characterized by a constant normal restitution coefficient $\alpha$. To achieve a stationary state, energy is supplied into the system by random forces acting on all particles. The random forces $\mathbf{F}_{i}$ are modeled as a white noise of intensity $\Gamma:\left\langle\mathbf{F}_{i}(t) \mathbf{F}_{k}\left(t^{\prime}\right)\right\rangle=m \Gamma \delta_{i k} \delta\left(t-t^{\prime}\right)$. This system reaches a homogeneous stationary state characterized by long-range correlations, leading to the renormalization of the energy density and collision frequency due to the fluctuations at low wave vectors [10].

Our system is composed of $N$ grains put in a square box with periodic boundary conditions. In addition to the small grains, two inelastic impenetrable and immobile large hard disks (the intruders) of diameter $D$ are placed, separated at a distance $R$. The coefficient of restitution $\alpha$ is the same for 
all types of collisions. This granular mixture is studied using molecular dynamics simulations. The grain-grain and grain-intruder collisions are treated as usual, using an event-driven code. To take into account the random forces, a new type of event is introduced: collisions between particles and a "thermal bath." This event results in a momentum $\mathbf{p}$ being instantaneously transferred to the particle, where $\mathbf{p}$ is randomly chosen by sampling a Gaussian distribution with zero mean and given variance $P_{\text {bath }}^{2}$ in each direction. Interactions with the thermal bath are scheduled for each grain. When an interaction takes place for a particle, its momentum gets updated and a new future event is scheduled for the same particle, after a random interval of time $t_{\text {next }}$, according to an exponential distribution $P\left(t_{\text {next }}\right) \sim \exp \left(-t_{\text {next }} / \tau_{\text {bath }}\right)$. In the limit $\tau_{\text {bath }} \rightarrow 0$ and $P_{\text {bath }} \rightarrow 0$, this injection method converges to the white noise force with $\Gamma=P_{\text {bath }}^{2} / m \tau_{\text {bath }}$. In practice, the time scale for interaction with the thermal bath, $\tau_{\text {bath }}$, is taken smaller than the free-flight time.

Hereafter, we choose as basic units $d, m$, and $\Gamma$. These units define the time unit as $t_{0}=\left(m d^{2} / \Gamma\right)^{1 / 3}$ and the energy unit as $e_{0}=\left(m d^{2} \Gamma^{2}\right)^{1 / 3}$. We take the diameter of the intruders $D=8 d$ and the coefficient of restitution $\alpha=$ 0.8 . We simulate systems of size $L=60 d$ and $L=80 d$, with the number density of the granular fluid $n=$ $0.366 d^{-2}$. Given the density, restitution coefficient, and noise intensity, the stationary temperature can be computed using mean field models giving $T_{0}=1.84 e_{0}$, and the collision frequency is $\nu_{0}=3.03 t_{0}^{-1}$. Hydrodynamic fluctuations determine a stationary temperature higher than $T_{0}$ that depends on the system size [10]. For $L=60 d, T=$ $2.43 e_{0}$ and for $L=80 \mathrm{~d}, T=2.46 e_{0}$. For every configuration, simulations were run for about $5 \times 10^{6}$ collisions per particle. We investigated the effective interaction between the intruders at a distance $R$. For this purpose, we measured the component of the total momentum transferred from the gas to intruders 1 and 2 along the line, parallel to the $x$ axis, joining their centers, $P_{i x}(i=1,2)$ as an average over a time interval $\tau=33.4 t_{0}$ (corresponding to about 100 collisions per particle). This procedure gives the "instantaneous" value of the fluctuating force as $F_{12}=$ $\left\langle P_{2 x}-P_{1 x}\right\rangle / 2 \tau$, whose time average finally leads to the net effective force $F$. In the elastic case, $\alpha=1, \Gamma=0, F$ vanishes as expected, whereas for $\alpha<1, \Gamma \neq 0, F$ is definitively different from zero, showing an effective force between intruders. The average $y$ component of the force is compatible with zero to numerical accuracy. On varying the noise strength, we checked that the force is proportional to the granular temperature, as can be deduced by dimensional analysis.

Figure 1 shows the relative force as a function of the distance $R$ for the two system sizes. The inset shows the short-range part of the relative force that extends for some small particle diameters $d$, alternating between attraction and repulsion. At larger distances, a repulsive force is observed with an interaction range much larger than $d$

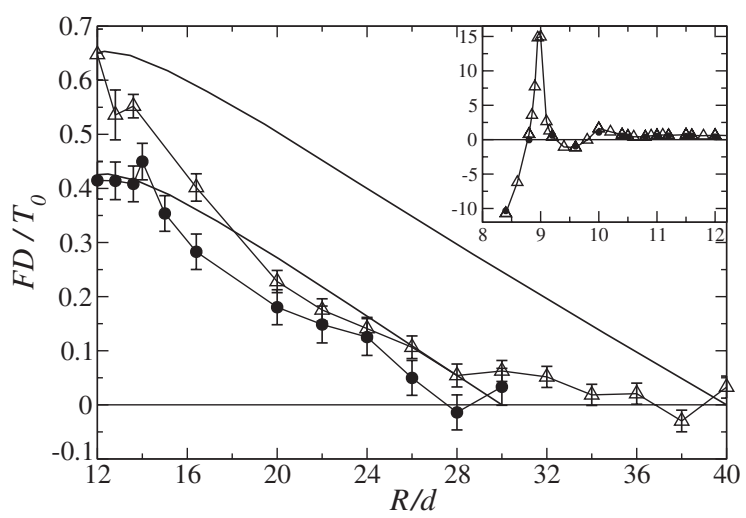

FIG. 1. Relative effective dimensionless force $F D / T_{0}$ between the intruders as a function of the distance $R$ between their centers. We plot simulations results for $L=60 d$ (solid circles) and for $L=80 d$ (open triangles), together with theoretical predictions (solid lines). Inset: Short-range region showing the depletion forces. Note the difference in the vertical scale, compared with the long-range part. Error bars in the inset are smaller than the symbols.

and comparable with $D$ or the box width (actually, due to the periodic boundary conditions, the force must vanish at $R=L / 2$, as seen from the simulations).

The oscillatory short-range part is similar to the depletion forces appearing in fluids that develop local layering structures [13,14], which are usually explained by entropic arguments based on equilibrium statistical mechanics. Recent works [6,15] suggest that depletion forces might be at work in granular mixtures, so the concept of entropic forces is applicable here. However, the long-range part cannot be due to a depletion mechanism as it extends beyond the typical range of the depletion forces and because it is repulsive in all its range.

To elucidate the nature of this force, we analyzed the probability distribution of the force over the intruders, plotted in Fig. 2. There it is seen that fluctuations are about 20 times larger than the average force, and therefore very long simulations are required. As the force is proportional to the granular temperature, these large fluctuations are not an artifact of a high temperature but an intrinsic property. Large fluctuations are a generic signature of fluctuationinduced forces (see, e.g., $[9,16])$.

This fact, together with the known property of large fluctuations in driven granular media, suggests that the long-range repulsion is a fluctuation-induced force as in the Casimir effect $[8,9]$. The confinement of the hydrodynamic fluctuations between intruders restricts the allowed fluctuation modes to those with wavelengths smaller than the gap size, whereas the spectrum of fluctuations in the outer region allows smaller wave vectors and forms a quasicontinuum, as illustrated in Fig. 3. Consequently, the hydrodynamically generated "radiation pressure" between the intruders is different from the one outside this inner region. 


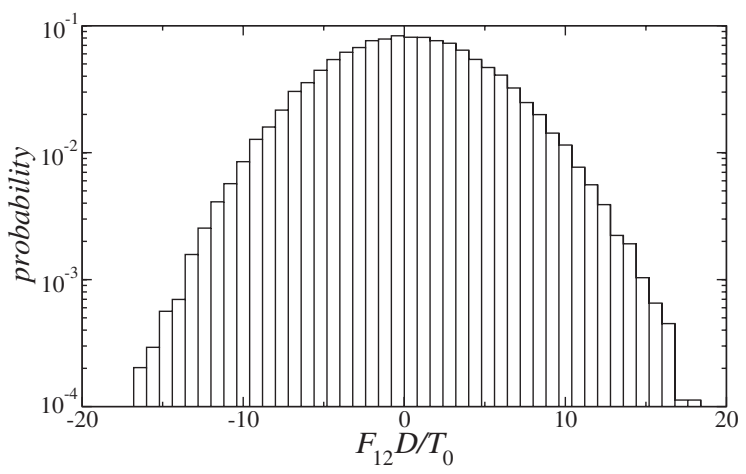

FIG. 2. Probability distribution of the fluctuating force $F_{12}$ at $R=20 d$, for $L=80 d$. The net effective force is obtained as the average of this histogram. The average value is $F=0.223 T_{0} / D$ and the standard deviation is $\sigma_{F}=4.83 T_{0} / D$, which is about 20 times larger than the average value.

To describe the Casimir force originated by the fluctuating hydrodynamic fields on the intruders, we use an approach similar to Ref. [10], where the pressure is renormalized in each point due to fluctuations that are computed using fluctuating hydrodynamics. Instantaneously, the pressure tensor at position $\mathbf{r}$ is given by

$$
p^{*}(\mathbf{r})=p(n(\mathbf{r}), T(\mathbf{r})) I+m n(\mathbf{r}) \mathbf{u}(\mathbf{r}) \mathbf{u}(\mathbf{r}),
$$

where $n, T$, and $\mathbf{u}$ are the fluctuating density, temperature, and velocity fields, respectively, and $I$ the identity tensor. Moreover, $p(n, T)=T H(n)$ is the usual thermodynamic pressure for hard disks [17], with $H(n)=n(1+$ $\left.\phi^{2} / 8\right) /(1-\phi)^{2}$ and $\phi=\pi n d^{2} / 4$. As the intruders are immobile, $\mathbf{u}$ vanishes at their surface, so the contribution of the convective term in $p^{*}$ vanishes and it becomes a scalar. This would not be the case if the intruders were allowed to move.

Linearizing the hydrodynamic fields $(n, T, \mathbf{u})$ around the stationary values $\left(n_{0}, T_{0}, 0\right)$, we can expand the pressure up to second order in the fluctuations $\delta n$ and $\delta T$. Its statistical average over the random noise is

$$
\left\langle p^{*}\right\rangle=p_{0}+H_{1}\langle\delta T \delta n\rangle+T_{0} H_{2}\left\langle\delta n^{2}\right\rangle,
$$

where $p_{0}=p\left(n_{0}, T_{0}\right), \quad H_{1}=d H /\left.d n\right|_{n_{0}}, \quad$ and $\quad H_{2}=$ $\frac{1}{2} d^{2} H /\left.d n^{2}\right|_{n_{0}}$. The density-density and densitytemperature fluctuations appearing in (2) are difficult to compute because they require solving the fluctuating hydrodynamic equations with the full boundary conditions imposed by the intruders. Alternatively, one could solve the corresponding equation for the correlation functions including the boundary conditions. A simpler estimation of $\left\langle p^{*}\right\rangle$ can be obtained by employing the Fourier transforms of the fluctuating fields $\delta A(\mathbf{r})=V^{-1} \sum_{\mathbf{k}} e^{-i \mathbf{k} \cdot \mathbf{r}} \delta A_{\mathbf{k}}$ and the structure factors $S_{A B}(\mathbf{k})=V^{-1}\left\langle\delta A_{\mathbf{k}} \delta B_{-\mathbf{k}}\right\rangle$ [10]. Expression (2) transforms into:

$$
\left\langle p^{*}\right\rangle=p_{0}+V^{-1} \sum_{\mathbf{k}}\left[H_{1} S_{n T}(\mathbf{k})+T_{0} H_{2} S_{n n}(\mathbf{k})\right] .
$$

The structure factors for the uniformly driven system have been described in detail in Ref. [10]. The relevant contribution comes from the region at small $k$, where they show a power law dependence $S_{A B}(\mathbf{k})=S_{A B}^{0} k^{-2}$. The prefactors $S_{A B}^{0}$ depend on density, noise intensity, and restitution coefficient $\alpha$. These asymptotic expressions, when inserted into (3), yield a sum $C \sum_{\mathbf{k}} k^{-2}$, where the coefficient $C \equiv H_{1} S_{n T}^{0}+T_{0} H_{2} S_{n n}^{0}$ turns out to be negative for $n<0.73 d^{-2}$ and positive for larger densities. Therefore, for $n<0.73 d^{-2}$ (the case of our simulations), fluctuations produce a decrease of the local pressure. In the gap between the intruders (region I according to Fig. 3), the number of possible $\mathbf{k}$ modes of low $k$ is smaller than outside (region II). The effect is that the pressure is lower outside than inside, leading to an effective repulsive force between the intruders. Furthermore, $C$ is proportional to the temperature; so is the Casimir force. The sign of the force is reversed for densities $n>0.73 d^{-2}$, which are too close to the freezing transition or random close packing [18] to be observed in the simulations. Experimentally, analogous crossover is found by increasing the driving intensity [7].

Because of the long-range correlations, fluctuations in regions I and II in Fig. 3 are correlated. However, in order to numerically estimate the $\mathbf{k}$ sums, we treat these regions as being independent. This approximation will overestimate the pressure difference and, hence, the Casimir force. However, it provides a rough estimation of its numerical value. In detail, we perform the $\mathbf{k}$ sum only over the $\mathbf{k}$ vectors allowed by the geometrical constraints. In a rectangular box of size $a \times b$, the $x$ component of the $\mathbf{k}$ vectors is $2 \pi n_{x} / a$ and the $y$ component is $2 \pi n_{y} / b$. It is at this point where the difference between regions I and II appears: $a=R-D$ in region $\mathrm{I}$ and $a=L-R-D$ in region II, while $b=D$ in both regions. Moreover, the vector $\mathbf{k}=(0,0)$ must be excluded from the sum, and we introduce an ultraviolet cutoff $k_{c}$, beyond which hydrodynamics is not valid. We take the cutoff $k_{c}=2 \pi / d_{0}$, where $d_{0}=\max \left(d, l_{0}\right)$ and $l_{0}$ is the mean free path of the small particles.

In the limit of small $k$, with structure factors going as $k^{-2}$, the pressure (3) can be analyzed asymptotically in the

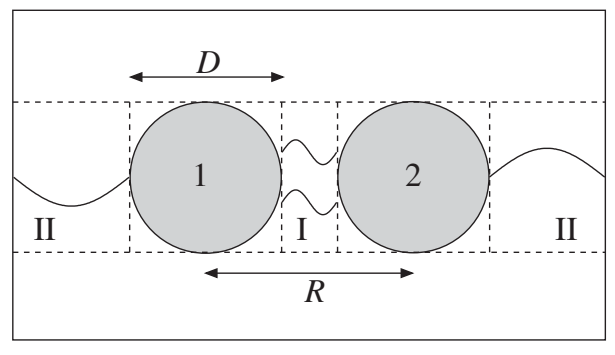

FIG. 3. Sketch of the hydrodynamic fluctuations leading to the Casimir-like force. In the gap between the intruders, only fluctuations of wavelength smaller than $R-D$ are allowed, while in the outer space the wavelengths can be larger. 
cases $a \gg b$ (for particles at large distances) and $a \ll b$ (for particles at short distances)

$$
\left\langle p^{*}\right\rangle= \begin{cases}p_{0}+C a / b ; & a \gg b \gg d_{0} \\ p_{0}+C b / a ; & b \gg a \gg d_{0} .\end{cases}
$$

These asymptotic expressions for the renormalized pressure do not depend on the cutoff distance, as long as $a$ and $b$ are much larger than it. Finally, the effective force on particle 2 is the difference of the forces at the left and the right of the particle $F_{2}=D\left[\left\langle p_{\mathrm{I}}^{*}\right\rangle-\left\langle p_{\mathrm{II}}^{*}\right\rangle\right]$. A negative value of $C$ gives rise to a long-range linear repulsive force and a short-range attractive force, at distances smaller than $D$. The opposite is obtained when $C$ is positive. Note that in this estimate the force depends on the system size. This fact is related to the structure of the fluctuations that become larger for small wave vectors. Therefore, when the system size is increased, at fixed $R$, the fluctuations in region II become larger, lowering the pressure in this region. However, the result (4) is only approximate at long distances, as mentioned.

The Casimir force can be computed numerically using the full expression of the structure factors [10], not only the $k^{-2}$ dominant term. The computed force is shown in Fig. 1. Note that the linear force dependence is preserved but the attractive part is lost, mainly because the simulation box is finite and corrections to the $k^{-2}$ order are observed. The computation using the $k^{-2}$ part shows a small difference of about $10 \%$ compared to the full calculation. The comparison with the simulations indicates that this prediction overestimates, as expected, the Casimir force, especially in the $L=80 d$ case, but it does give the same order of magnitude and correct sign of the force. Moreover, the predicted force for $L=80 d$ is larger than for $L=60 d$, in agreement with the simulations. However, our theory does not predict the saturation of the force observed for $R \geqslant 20 d$. Possible sources for this discrepancy are (a) hydrodynamic correlations between regions I and II reducing the pressure difference, (b) geometrical factors that arise from considering rectangular regions instead of those bounded by circles, and (c) fixed intruders that break the Galilean invariance and may modify the structure factors at very short wave vectors.

To summarize, we have found that two intruders, immersed in a sea of smaller granular particles driven by a white noise force, experience a long-range mutual repulsion. This repulsion has a dynamical origin and cannot be explained by standard depletion forces. We have proposed a mechanism based on the confinement of hydrodynamic fluctuations when the intruders are near. The present effect is an example of repulsion determined by fluctuationinduced forces instead of the standard attraction, a phenomenon which has been predicted to occur also in polymers [19].

We claim that the force we observe is the granular analog of the Casimir effect. We propose a novel method, valid for nonequilibrium fluids, to compute Casimir forces starting from the structure factors. The two key ingredients which render this effect manifest in the context of granular gases are (a) the occurrence of large low- $k$ fluctuations, originated in the coupling of nonconserving noise with conserving hydrodynamic fields (conservation of particle number and momentum in collisions) [10] and (b) the confinement of these fluctuations in a gap (the space between the intruders), which is the common feature of all instances of Casimir forces. The enhancement of the low- $k$ components plays the role of criticality in equilibrium molecular systems, where forces are induced by the thermal fluctuations of a correlated fluid in a confining geometry. Finally, these long-range forces might be responsible for segregation effects in vibrofluidized granular mixtures of particles having different material properties.

We acknowledge partial support from RIKEN. C. C. and F. N. acknowledge JSPS, NSA, and ARDA under AFOSR Contract No. F49620-02-1-0334 and by NSF Grant No. EIA-0130383. R. B. is supported by Projects No. FIS04-271 (Spain) and No. UCM PR27/05-13923BSCH. U.M. B. M. is financed by Grant Cofin-Miur 2005, No. 2005027808. R.S. is partly financed by Fondecyt (Grant No. 1030993), Fondap (No. 11980002) and Universidad Complutense (Profesores Visitantes).

[1] H. M. Jaeger et al., Rev. Mod. Phys. 68, 1259 (1996); J. Duran, Sands, Powders, and Grains (Springer, New York, 2000).

[2] A. Kudrolli, Rep. Prog. Phys. 67, 209 (2004).

[3] S. J. Moon et al., Phys. Rev. Lett. 91, 134301 (2003); T. Schnautz et al., Phys. Rev. Lett. 95, 028001 (2005).

[4] C. Cattuto and U. Marini Bettolo Marconi, Phys. Rev. Lett. 92, 174502 (2004).

[5] S. Aumaitre, C. A. Kruelle, and I. Rehberg, Phys. Rev. E 64, 041305 (2001).

[6] D. A. Sanders et al., Phys. Rev. Lett. 93, 208002 (2004).

[7] I. Zuriguel et al., Phys. Rev. Lett. 95, 258002 (2005).

[8] H.B. G. Casimir, Proc. K. Ned. Akad. Wet. 51, 793 (1948).

[9] M. Kardar and R. Golestanian, Rev. Mod. Phys. 71, 1233 (1999).

[10] T. P. C. van Noije et al., Phys. Rev. E 59, 4326 (1999).

[11] M. Bretz et al., Phys. Rev. Lett. 69, 2431 (1992).

[12] G. Peng and T. Ohta, Phys. Rev. E 58, 4737 (1998).

[13] B. Gotzelmann, R. Evans, and S. Dietrich, Phys. Rev. E 57, 6785 (1998).

[14] J. C. Crocker et al., Phys. Rev. Lett. 82, 4352 (1999).

[15] M. Bose et al., Phys. Rev. E 72, 021305 (2005).

[16] D. Bartolo et al., Phys. Rev. Lett. 89, 230601 (2002).

[17] L. Verlet and D. Levesque, Mol. Phys. 46, 969 (1982).

[18] C. F. Tejero and J. A. Cuesta, Phys. Rev. E 47, 490 (1993).

[19] S. P. Obukhov and A. N. Semenov, Phys. Rev. Lett. 95, 038305 (2005). 